\title{
Salvage of an Osteocutaneous Fibula Flap with a Variant Perforator of Skin Paddle in Lower Leg Reconstruction
}

\author{
Kenji Kawamura, MD ${ }^{1}$ Shohei Omokawa, MD ${ }^{1}$ Takamasa Shimizu, MD ${ }^{1}$ Tadanobu Onishi, MD \\ Satoshi Hayashi, MD ${ }^{1}$ Naoki Maegawa, MD ${ }^{1}$ Yasuhito Tanaka, MD ${ }^{1}$
} ${ }^{1}$ Department of Orthopaedic Surgery, Nara Medical University,
Nara, Japan

J Reconstr Microsurg Open 2017;2:e90-e93.
Address for correspondence Kenji Kawamura, MD, Department of Orthopaedic Surgery, Nara Medical University, 840 Shijo-Cho, Kashihara, Nara 634-8522, Japan (e-mail: kkenji@naramed-u.ac.jp).

\begin{abstract}
Background The osteocutaneous fibula flap is an established method for reconstruction of bone and soft tissue defects in the lower extremity. The vascularity of the fibula and overlying skin paddle is usually provided by a single pedicle composed of the peroneal artery. In rare situations, the fibula is supplied by the peroneal artery, whereas the overlying skin paddle is supplied by perforators originating from the posterior tibial artery.

Case Report A 28-year-old man presented with osteomyelitis of the tibia that was scheduled to be treated with a free vascularized osteocutaneous fibula flap from the contralateral lower leg. Intraoperatively, it was found that perforators supplying the skin paddle originated not from the peroneal artery but from the posterior tibial artery. A fibula flap nourished by the peroneal vessels was harvested and the skin paddle was returned to the lower leg. The fibula was fixed at the recipient site, and peroneal vessels were anastomosed to the recipient posterior tibial vessels. The skin defect was successfully managed with a perforator-based propeller flap nourished by the recipient artery.

\section{Keywords}

- osteocutaneous fibula flap

- variant perforator

- lower leg reconstruction

Conclusion To the best of our knowledge, only five authors have reported this variant vascularity of the osteocutaneous fibula flap. They harvested two independent flaps, one a skin flap and the other a fibula flap, and performed two separate vascular anastomoses at the recipient site. In comparison to previously reported cases, the salvage procedure using a perforator-based propeller flap is easy and reliable because there is no need for additional anastomosis of the perforator vessels.
\end{abstract}

The vascularized free osteocutaneous fibula flap has become a universal technique for reconstruction of bone and soft tissue defects in the lower leg since Taylor et al first described it in $1975 .^{1}$ The fibula and overlying skin paddle are usually nourished by a single pedicle composed of the peroneal artery and comitant veins. However, to the best of our knowledge, five authors have described a rare variant where the fibula was supplied by the peroneal artery, and the overlying skin paddle was supplied by the perforators originating from the posterior tibial artery. ${ }^{2-6}$ In such rare situations, the fibula and skin flaps would have to be revascularized separately and the skin paddle cannot serve as a monitor for the fibula. We present a rare variant encountered while elevating an osteocutaneous fibula flap received

June 1, 2017

accepted

June 11, 2017
DOI https://doi.org/

$10.1055 / \mathrm{s}-0037-1604340$.

ISSN 2377-0813.
Copyright $\odot 2017$ by Thieme Medical

Publishers, Inc., 333 Seventh Avenue, New York, NY 10001, USA. Tel: +1(212) 584-4662.
License terms

(요 (1) $\circledast$ 
for reconstruction of an infected nonunion of the contralateral tibia and successful management with a perforatorbased propeller flap nourished by the recipient artery.

\section{Case Report}

A 28-year-old man presented with osteomyelitis of the left tibia due to a 12-month methicillin-resistant Staphylococcus aureus (MRSA) infection, which occurred after he sustained fractures of left distal tibia and fibula in an accidental fall (-Fig.1). A free vascularized osteocutaneous fibula flap from the contralateral lower leg was planned to reconstruct the bone and soft tissue defects. Preoperative computed tomography (CT) angiography of the lower legs revealed no vascular anomalies in the donor right leg and obstruction of the peroneal artery in the injured left leg. The perforators in the distal third of the right lower leg were marked using a Doppler rheometer. Intraoperatively, the two septocutaneous perforators within the posterior crural septum were identified. These perforators ran between the soleus muscle and flexor hallucis longus muscle and then joined the posterior tibial artery (-Fig. 2). After discovery of the rare variant anatomy in the donor leg, the posterior tibial artery and comitant veins were dissected as the recipient vessels in the injured leg. We found a large septocutaneous perforator originating from the recipient posterior tibial artery, which was used for harvesting a perforator-based propeller flap to cover the skin defect. A fibula flap nourished by the peroneal artery and comitant veins was harvested, and the skin paddle
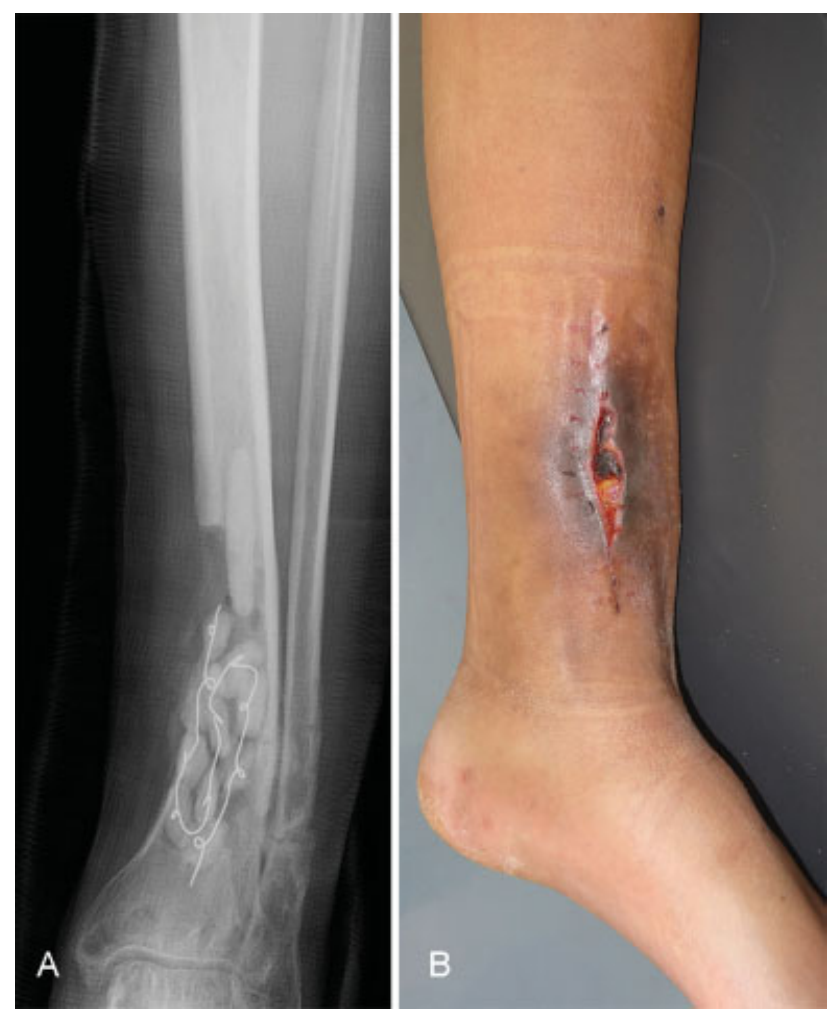

Fig. 1 Preoperative findings. (A) Preoperative $X$-ray shows nonunion of the left tibia. There are antibiotic-loaded cement beads in the bone defect site. (B) A skin defect at the nonunion site.

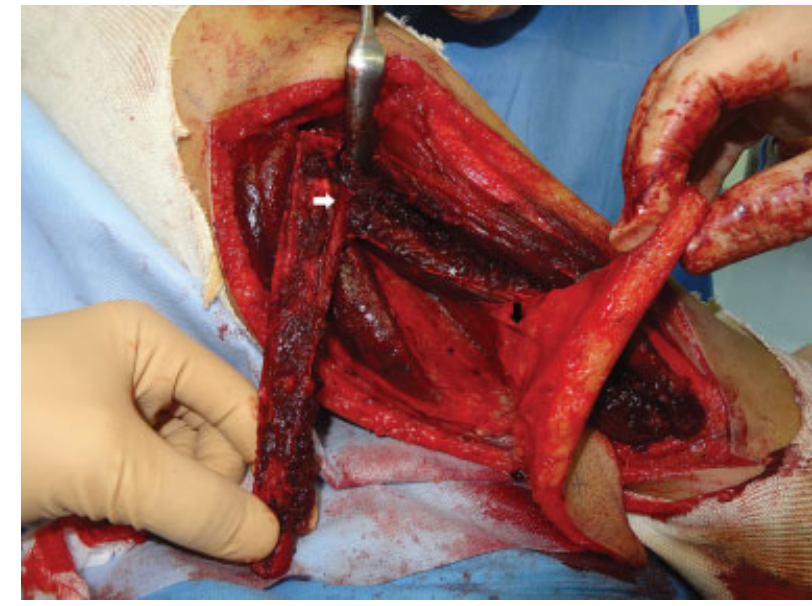

Fig. 2 Intraoperative findings of the donor leg. Intraoperative photograph shows that the fibula is supplied by the peroneal artery (white arrow) and the skin paddle is supplied by the perforators originating from the posterior tibial artery (black arrow). The perforator runs between the soleus muscle (black asterisk) and the flexor hallucis longus muscle (white asterisk) and finally joins the posterior tibial artery.

was returned to the lower leg. After fixing the fibula at the recipient site, the peroneal artery of the fibula flap was anastomosed to the posterior tibial artery at the distal site of the perforator origin. The comitant veins of the peroneal artery were anastomosed to the comitant veins of the posterior tibial artery. Then, the skin defect was covered with a posterior tibial artery-based perforator propeller flap (-Fig. 3). Two weeks later, an additional skin graft was applied to the donor site of the propeller flap (-Fig. 4).

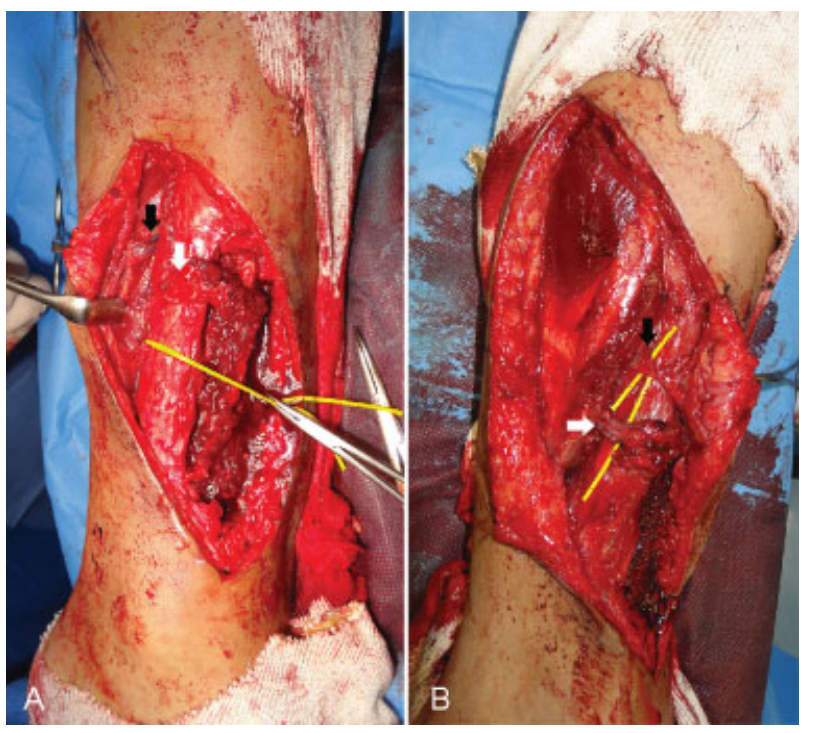

Fig. 3 (A) Intraoperative findings of the recipient leg. After fixing the fibula at the recipient site, there is a large septocutaneous perforator originating from the posterior tibial artery (black arrow). White arrow indicates vascular pedicle of the fibula flap composed of peroneal vessels. (B) After harvesting a propeller flap nourished by a perforator originating from the posterior tibial artery (black arrow), the peroneal vessels of the fibula flap are anastomosed end-to-end to the posterior tibial vessels at the distal site of the perforator origin (white arrow). 


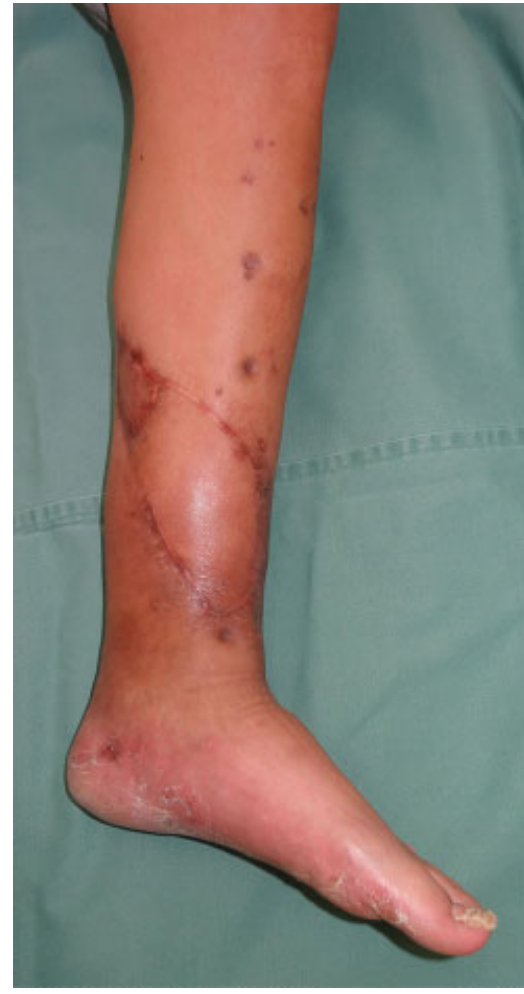

Fig. 4 Postoperative appearance. The skin defect heals well after an additional skin graft to the donor site of the propeller flap.

The postoperative course was uneventful. Four months after the operation, the patient began walking without assistance, and an X-ray of the left lower leg showed good union between the grafted fibula and the recipient tibia (- Fig. 5).

\section{Discussion}

Since Taylor et al first reported the successful use of a free vascularized fibula graft for reconstruction of a tibial bone defect, various anatomic studies have been conducted to improve the surgical procedure of the osteocutaneous fibula flap. ${ }^{1}$ It has been reported that there are between three and eight perforators in the lateral lower leg. ${ }^{7,8}$ The vast majority of these perforators arise from the peroneal artery, but it has been observed that the most proximal perforator may occasionally arise from the posterior tibial artery or tibialperoneal trunk. ${ }^{9}$ Therefore, raising a skin paddle from the distal third of the lateral lower leg has been recommended. Furthermore, most perforators in the distal third of the lateral lower leg are septocutaneous perforators, making dissection easier than the musculocutaneous perforators in the proximal lateral lower leg. In 2001, Yokoo et al first reported a case in the literature, whereby a septocutaneous perforator in the distal third of the lateral lower leg joined the posterior tibial artery. ${ }^{2}$ In such circumstances, the procedure has to be either abandoned or modified so that neither the vascularity of the flap nor the donor leg is compromised. They harvested two independent flaps, one a skin flap and second a fibula flap, and performed two

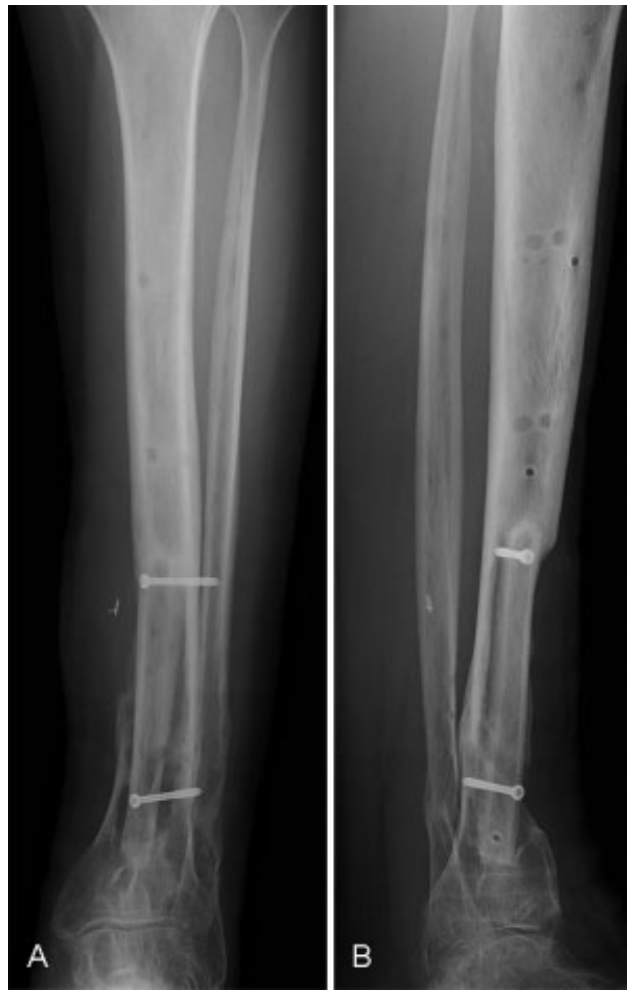

Fig. 5 Postoperative $X$-rays. Four months postoperatively, AP (A) and lateral (B) X-rays show good union between the grafted fibula and the recipient tibia. AP; anteroposterior.

separate vascular anastomoses at the recipient site for reconstruction of the mandible with the fibula flap and the lower gingiva with the skin flap. In 2009, Yadav et al also reported this variant perforator in the reconstruction of the mandible. ${ }^{3}$ They harvested the flap as a single composite and managed it with two separate vascular anastomoses at the recipient site. Tan and Wong reported the same variant perforator in the reconstruction of a comminuted fracture of the mandible. ${ }^{4}$ They abandoned harvesting the skin flap, and the oral laceration was repaired primarily and the reconstruction was achieved with bone only. Parr et al harvested two independent flaps in the same situation for reconstruction of the mandible. ${ }^{5}$ The peroneal vessels supplying the fibula flap were anastomosed to the recipient vessels, and the perforator vessels supplying the skin flap were anastomosed end-to-end to the distal ends of the peroneal vessels. Nakazawa et al reported the same variant perforator in the reconstruction of the lower leg. ${ }^{6}$ They harvested two independent flaps; the peroneal artery of the fibula flap was anastomosed end-to-end to the dorsalis pedis artery, and the perforator artery of the skin flap was anastomosed end-to-side to it. In our case, the bone defect was reconstructed with the fibula flap, and the skin defect was reconstructed with the perforator-based propeller flap nourished by the recipient artery, because additional anastomosis of the small-diameter perforator vessels is a complicated process. Recently, various perforator flaps that can preserve main arteries in the extremities have been developed. ${ }^{10}$ Surgeons should consider whether perforator flaps 
can be used for coverage of the skin defect when they encounter vascular variants during a osteocutaneous fibula flap procedure.

\section{Conclusion}

In comparison to previously reported cases, a salvage procedure with a perforator-based propeller flap nourished by a recipient artery is easy and reliable because there is no need for additional anastomoses.

\section{Conflict of Interest}

None.

\section{References}

1 Taylor GI, Miller GD, Ham FJ. The free vascularized bone graft. A clinical extension of microvascular techniques. Plast Reconstr Surg 1975;55(05):533-544

2 Yokoo S, Komori T, Furudoi S, Umeda M, Nomura T, Tahara S. Rare variant of the intrasoleus musculocutaneous perforator: clinical considerations in raising a free peroneal osteocutaneous flap. J Reconstr Microsurg 2001;17(04):225-228
3 Yadav PS, Ahmad QG, Shankhdhar VK, Nambi GI. Successful management of free osteocutaneous fibula flap with anomalous vascularity of the skin paddle. Indian J Plast Surg 2009;42(02): 255-257

4 Tan BK, Wong CH. An anomalous septocutaneous perforator to the skin paddle of the fibula osteocutaneous flap originating from the posterior tibial artery. J Plast Reconstr Aesthet Surg 2009;62(05): 690-692

5 Parr JM, Adams BM, Wagels M. Flow-through flap for salvage of fibula osseocutaneous vascular variations: a surgical approach and proposed modification of its classification. J Oral Maxillofac Surg 2014;72(06):1197-1202

6 Nakazawa H, Nozaki M, Higasimori T, et al. Fibula osteoseptocutaneous flap with a variant perforator and peroneal artery arising from the anterior tibial artery. J Reconstr Microsurg 2005;21(02): 119-124

7 Chen YL, Zheng BG, Zhu JM, et al. Microsurgical anatomy of the lateral skin flap of the leg. Ann Plast Surg 1985;15(04):313-318

8 Beppu M, Hanel DP, Johnston GH, Carmo JM, Tsai TM. The osteocutaneous fibula flap: an anatomic study. J Reconstr Microsurg 1992;8(03):215-223

9 Yajima $\mathrm{H}$, Ishida $\mathrm{H}$, Tamai S. Proximal lateral leg flap transfer utilizing major nutrient vessels to the soleus muscle. Plast Reconstr Surg 1994;93(07):1442-1448

10 Gir P, Cheng A, Oni G, Mojallal A, Saint-Cyr M. Pedicled-perforator (propeller) flaps in lower extremity defects: a systematic review. J Reconstr Microsurg 2012;28(09):595-601 